\title{
Modelo de Salud y Seguridad en el Trabajo con Gestión Integral para la Sustentabilidad de las organizaciones (SSeTGIS)
}

\author{
HEALTH AND SAFETY AT WORK MODEL WITH INTEGRAL MANAGEMENT FOR SUSTAINABILITY OF \\ ORGANIZATIONS (SSETGIS)
}

Ana Anaya-Velasco ${ }^{1}$

1. Departamento de Promoción, Preservación y Desarrollo de la Salud del Centro Universitario del Sur de la Universidad de Guadalajara.

\section{RESUMEN}

Ante los desafíos que plantea hacer a las empresas sustentables con responsabilidad social, es necesario desarrollar procesos de mejora basados en una gestión integral con impactos medibles en los resultados de calidad, productividad, salud y seguridad en el trabajo, el cuidado del medio ambiente y la cultura de protección civil. Para estas materias existen lineamientos internacionales y marcos jurídicos nacionales que cumplir, los cuales establecen la metodología para reconocer, evaluar y controlar los factores de riesgo de todo tipo en el lugar de trabajo y para la comunidad. Este documento presenta un modelo de gestión integral para la sustentabilidad de las organizaciones desde la perspectiva de la salud y la seguridad en el trabajo sin limitarse a ellas: se denomina SSeTGIS; es holístico, multidisciplinar, centrado en las personas, preferentemente participativo, el cual se desarrolla como un proceso de ciclos de mejora continua en tres niveles de actuación: individual, lugar de trabajo y organizacional. Se exponen los argumentos, los propósitos, los componentes, el proceso y la metodología, así como algunas notas de su aplicación.

(Anaya-Velasco A, 2017. Modelo de Salud y Seguridad en el Trabajo con Gestión Integral para la Sustentabilidad de las organizaciones (SSeTGIS). Cienc Trab. May-Ago; 19 [59]: 95-104).

Palabras claves: MODELO, SALUD, SEGURIDAD EN EL TRABAJO, GESTIÓN INTEGRAL, SUSTENTABILIDAD DE LAS ORGANIZACIONES.

\section{ABSTRACT}

Faced with the challenges of making sustainable companies with social responsibility, it is necessary to develop improved processes based on comprehensive management with measurable impacts on the results of quality, productivity, health and safety at work, environmental care and culture of civil protection. These subjects for which international guidelines and national legal frameworks exist for their fulfillment, establish the methodology for recognizing, evaluating and controlling all kinds of risk factors in the workplace and for the community. This document presents a model of integral management for the sustainability of organizations from the perspective of health and safety at work without limiting to them, referred to as SSeTGIS. It is holistic, multidisciplinary, people-centered, and preferably participatory. It is developed as a process of cycles of continuous improvement in three levels of performance: individual, workplace and organizational. The arguments, the purposes, the components, the process and the methodology are presented as well as some notes of their application.

Key words: MODEL, HEALTH, SAFETY, INTEGRAL MANAGEMENT, SUSTAINABILITY OF ORGANIZATIONS.

\section{INTRODUCCIÓN}

La salud y la seguridad de las personas son fundamentales para lograr una vida plena, un mejor desempeño laboral y el desarrollo de potencialidades en diferentes aspectos y ámbitos de su vida. Por ello, y para protegerlas, existen convenios internacionales y marcos jurídicos nacionales que recuperan los avances científicos y tecnológicos

Correspondencia / Correspondence:

Libramiento oriente 97 int. 3. C.P. 49064. Cd. Guzmán, Jalisco, México.

Tel.: +52 3414136747

e-mail, contacto: anayav@cusur.udg.mx

e-mail:velasana_@hotmail.com

Recibido: 14 de Marzo de 2017 / Aceptado: 29 de Junio de 2017 en materia de salud, seguridad e higiene en el trabajo, cuidado del ambiente y protección civil.

En contraposición a los beneficios de la gestión integral de las organizaciones con perspectiva de salud y seguridad en el trabajo, los daños a las personas crecen a pasos alarmantes.

La Organización Internacional del Trabajo (OIT) en 2014 "calculó que los accidentes de trabajo y las enfermedades profesionales causan más de 2.3 millones de muertes anuales, de la cuales más de 350.000 son por accidentes de trabajo, y aproximadamente 2 millones son por enfermedades profesionales"1; agrega que "estas cifras, aunque sorprendentes, no expresan el total de las pérdidas económicas de las empresas y sociedades"1 ${ }^{1}$, tampoco el padecimiento físico y emocional de los trabajadores y sus familias. Esto plantea desafíos de calidad y productividad $^{2}$ asociados a las condiciones del ambiente físico, psicosocial y de la organización del trabajo, así como de su entorno. Por otro lado, la Organización de las Naciones Unidas ${ }^{3}$, estableció los 17 objetivos de desarrollo sostenible, que muestran la necesidad del 
cuidado del medio ambiente y la atención a las necesidades humanas clasificadas por Max-Neef ${ }^{4}$, así como el tipo de sociedad y las formas de producción sostenibles. Asimismo, propone a los paises una planeación integral para alcanzarlos en 2030.

La propia Organización de Naciones Unidas en su informe de Evaluación global sobre la reducción de riesgos de desastres ${ }^{5}$, indica que, entre 1980 y 2012, cada año se perdieron 42 millones de años de vida humana (concepto utilizado por la misma organización) en desastres tales como terremotos, tsunamis, ciclones e inundaciones con pérdidas económicas entre 250.000 millones y 300.000 millones de dólares americanos al año. Señala que es necesario "gestionar los riesgos en lugar de gestionar desastres: la reducción de riesgos debe reinterpretarse." ${ }^{5}$

No obstante estos datos e iniciativas, es difícil para los directivos y el personal de las organizaciones comprender la necesidad y los beneficios de una gestión integral de la salud, la seguridad, el cuidado del ambiente, la calidad y la productividad como una cultura de gestión y de trabajo para tratarlas al mismo nivel y en sinergia, no como adición sino como unificación en las políticas, los objetivos y la actuación para aprovechar al máximo los recursos a favor de la sustentabilidad. Así lo presentan modelos integrales como el de Responsabilidad social de la International Standard Organization ${ }^{6}$ y el de la Organización Mundial de la Salud7; incluso en tiempos de crisis; y los propuestos por Salanova ${ }^{8}$ y Neira, quienes señalan: "La riqueza de las empresas depende de la salud de los trabajadores". También se dificulta entender la necesidad de contar con programas, un responsable competente para coordinar y asesorar en esas áreas, la estructura organizacional que le dé soporte a su gestión y un presupuesto para operar ${ }^{10-11}$.

Parte de esa dificultad se debe a la multiplicidad de factores de riesgo de diferente naturaleza, ya sea química, física, biológica, psicosocial, ergonómica, mecánica, eléctrica, estructural o natural, que deriva de la actividad de la organización y su proceso productivo del bien o servicio, y del lugar donde se desarrollan, lo cual implica la multi-, trans- e interdisciplina. Dichos factores son contemplados en el marco jurídico construido como sociedad para la protección de los derechos humanos, laborales y del ambiente; y es obligatorio para las organizaciones y los trabajadores, para las instituciones, los ciudadanos y el gobierno.

La salud laboral es, en primer lugar, una preocupación y responsabilidad de las propias personas involucradas en el trabajo, vale decir trabajadores y empleadores. No es ético que las personas malogren su salud intentando ganarse la vida. ${ }^{12}$

Ante los datos presentados, es importante que las organizaciones cuenten con alternativas de modelos de gestión integral para lograr sus objetivos con beneficios compartidos y medibles, a través de los cuales los trabajadores puedan acceder a los satisfactores de sus necesidades humanas fundamentales, al tiempo que contribuyen a resultados favorables para la organización.

Aquí se plantea el modelo SSeTGIS para la sustentabilidad de las organizaciones, con trabajo decente y responsabilidad social. Se presentan los antecedentes del modelo y sus propósitos; el modelo, los componentes, sus requerimientos en cuanto a políticas, responsable y presupuesto; el proceso, la metodología para reconocer, evaluar y controlar los factores de riesgo, y los niveles de actuación.

\section{Antecedentes del modelo SSeTGIS}

A partir de la necesidad de abordar el estudio y la aplicación de procesos de mejora en las organizaciones basados en una gestión integral para la calidad de vida en el trabajo con impactos favora- bles en indicadores de salud y seguridad del personal, cuidado del medio ambiente, la protección civil (SSeTMAPC) y en los resultados de la organización en cuanto a calidad y productividad, y como producto de la línea de investigación "Salud y seguridad en el trabajo con gestión integral para la sustentabilidad de las organizaciones" a la cual se dedica, la autora creó el modelo de gestión integral SSeTGIS (léase "setgis"), que considera a las personas en su entorno de trabajo como la base y las primeras beneficiarias del proceso investigativo al tiempo que genera conocimientos para una mayor comprensión del proceso de mejora de la organización. Un propósito de dicha línea fue la formación de recursos humanos como profesionales competentes en la prevención, atención, gestión e investigación de factores de riesgo, lo cual expandió la gestión e investigación en esos campos en las organizaciones en las que intervienen y sobre la necesidad de sistemas integrales.

El marco teórico del modelo está compuesto principalmente por los lineamientos internacionales y por cada marco jurídico nacional de salud y de seguridad e higiene en el trabajo como perspectiva, también por el de medio ambiente y el de protección civil; además de la teoría general de sistemas ${ }^{13}$, que permite la integración de aquellas áreas disciplinares entre sí y con la calidad y la productividad. Así lo proponen los sistemas de gestión integral involucrados en la responsabilidad social ${ }^{6}$, en entornos laborales saludables ${ }^{7} \mathrm{y}$ las organizaciones saludables $\mathrm{y}$ resilientes. ${ }^{14}$

De igual manera, toma como referente para la mejora el enfoque de la investigación-acción de ciclos multifases de procesos participativos, las teorías de la psicología del trabajo y las organizaciones, de la gestión del talento humano, y de las necesidades humanas. ${ }^{4}$

Los modelos de gestión integral como el SSeTGIS son una opción para dar cumplimiento a la misión y los objetivos de las organizaciones.

Los objetivos de integrar los sistemas de gestión son: identificar, manejar y reducir los efectos de producto no conforme; reducir los efectos ambientales y los peligros de actividades de los trabajadores; capacitar al personal y asegurar su participación para la mejora continua del desempeño de la calidad; fomentar la seguridad y cuidar el medio ambiente, entre otras. ${ }^{15}$

Cabe mencionar que las empresas e instituciones destacadas por su cultura y sus resultados cuentan en su estructura organizacional con un área de primer nivel identificada como de Salud, Seguridad y Medio Ambiente (EHS por sus siglas en inglés [Environmental, Health and Safety]) y con una política especifica en esas materias. ${ }^{6-7}$

\section{El modelo}

El modelo SSeTGIS es holístico, con gestión integral de las organizaciones desde la perspectiva de la salud y la seguridad en el trabajo, sin limitarse a ellas, considera el cuidado del entorno natural y social, con calidad y productividad. El modelo SSeTGIS está compuesto por las diferentes condiciones ${ }^{16}$ y factores de riesgo ${ }^{17}$ de cada una de las áreas que gestiona además de los indicadores ${ }^{18}$ de calidad y productividad.

\section{Propósitos}

Promover estilos de vida saludable entre todos los trabajadores y las trabajadoras hacia la sustentabilidad; mejorar las condiciones de trabajo y el cuidado del ambiente con calidad y productividad; emplear la gestión integral centrada en las personas como cultura 
organizacional con respeto a los derechos, valorando la participación y el desarrollo del potencial humano, y proyectar estos principios de trabajo decente y responsabilidad social hacia sus familias, los destinatarios del bien o servicio, las demás partes interesadas y la sociedad.

Tiene como perspectiva la salud y seguridad en el trabajo, ya que los lugares de trabajo y las personas que lo realizan coinciden con el origen de los riesgos potenciales no sólo a la salud, al ambiente y a la comunidad, sino también a la calidad y la productividad, debido a lo cual es posible, en ese mismo origen, iniciar ciclos de mejora continua como estrategia. Esto implica un proceso, unas políticas, la formación y la participación del personal de todos los niveles para interiorizar esta filosofía como cultura de trabajo.

Con esa perspectiva y cultura, cada persona se responsabiliza de su bienestar y cada encargado de área realiza su planeación estratégica y programa operativo interiorizando los costos y midiendo los beneficios; de tal manera que resulta mínimo el presupuesto necesario del individuo o la estructura organizacional responsable de coordinar, asesorar y documentar en esas materias en términos del marco jurídico aplicable. Para documentar, es conveniente tener un sistema de información.

\section{Componentes}

Los componentes dependen de la naturaleza y la actividad de la organización; pueden ser los siguientes:

- En salud, su promoción para una cultura de autocuidado a través de campañas de comunicación y programas específicos; su vigilancia debe considerar indicadores como los días laborados sobre los totales, el registro e investigación de los riesgos de trabajo y los exámenes médicos de ingreso, periódicos y específicos necesarios de acuerdo con la exposición, según la $\mathrm{OIT}^{19}$; además del perfil epidemiológico de la población. Es importante una evaluación de salud integral -biopsicosocial-.

- Entre las condiciones de higiene en el trabajo se encuentran los agentes ambientales químicos, biológicos y físicos (ruido, iluminación, condiciones térmicas elevadas o abatidas, radiaciones ionizantes y no ionizantes y vibraciones), los cuales pueden causar enfermedades laborales al personal ocupacionalmente expuesto y, de salir del lugar de trabajo, a la comunidad y contaminación al ambiente. También existen los factores de riesgo ergonómicos $^{20}$ y psicosociales. $^{21}$

- Dentro de las condiciones de seguridad en el trabajo se encuentran las de los dispositivos mecánicos y eléctricos, los edificios, los métodos de trabajo inseguros que pueden causar accidentes laborales, las instalaciones peligrosas relacionadas con sustancias químicas cuyo potencial de incendio, explosión o toxicidad las hace capaces de afectar a las personas, las instalaciones, el ambiente y, eventualmente, la sustentabilidad del propio centro de trabajo. ${ }^{1}$ La seguridad patrimonial es otro aspecto a considerar como el conjunto de normas y medidas que protegen los bienes y activos, patrimonio o haber de la organización.

- Los rubros del cuidado del medio ambiente, de acuerdo con los objetivos para el desarrollo sostenible de Naciones Unidas ${ }^{3}$, comprenden, entre otros: suelo y subsuelo, impacto ambiental, recursos naturales, abastecimiento de agua de pozos o de red municipal, descarga de agua, residuos de manejo especial, residuos peligrosos, emisiones a la atmósfera, riesgo ambiental, y ahorro de energía.

- La protección civil, por su parte, en algunas legislaciones nacionales, comprende la acción solidaria y participativa, que en consi- deración de los riesgos de origen natural o antrópico y sus efectos, prevé la coordinación de los sectores público, privado y social privilegiando la gestión integral de riesgos, para que se apliquen las medidas para salvaguardar la vida, integridad y salud de la población, la planta productiva y el medio ambiente. ${ }^{22}$

- Respecto la calidad y la productividad como gestión integral, el modelo contempla monitorear los indicadores de interés seleccionados por la organización de acuerdo con su actividad, a fin de constatar mediante la metodología adecuada que se mantienen o mejoran durante el proceso previsto por el modelo, el cual considera la sustentabilidad económica.

\section{Politicas y lineamientos}

El modelo SSeTGIS como sistema de gestión requiere primordialmente del compromiso de la dirección, de políticas y los lineamientos en SSeTMAPC; con planeación estratégica establecer los objetivos, estrategias y metas a alcanzar para todo el personal.

De no contar con ellos, es necesario proponerlos o elaborarlos en un taller con la dirección de la organización, en la que manifieste su compromiso con la visión y la forma de trabajo como filosofía de integralidad centrada en las personas que el modelo SSeTGIS plantea como propósitos de desarrollo y sustentabilidad, cuyos impactos puedan valorarse, percibirse y medirse.

La misión se redacta en términos de ser una organización que promueve estilos de vida saludables y seguros para todos, responsable del entorno físico y organizacional, del cuidado del ambiente y de la protección civil con gestión integral para lograr sus objetivos con la calidad y productividad que le dé sustentabilidad.

La visión contempla instalaciones seguras y agradables para la realización saludable de las actividades y el desarrollo integral del personal y los visitantes, en el que se cumplen los principios de trabajo decente a través de una gestión integral para la sustentabilidad, por lo cual se convierte en un entorno de aprendizaje para el cuidado del ambiente, la protección civil y la responsabilidad social.

Para hacer operativa la visión del modelo persona por persona, se plantea una "pausa SSeTGIS" a manera de filtro de análisis para la acción, como sigue:

Esta decisión o acción, es favorable a: $\mathrm{Si}$

- Mi salud integral -biopsicosocial.

- La seguridad mía y la de los demás.

- El cuidado del medio ambiente.

- La cultura de protección civil.

- La calidad de los procesos y los productos (o servicios).

- La productividad (consumo mínimo de recursos ahora o en el futuro).

De resultar favorable en todas las áreas analizadas, el trabajador o equipo de trabajo, procede con su decisión y acción. Si se encuentra que para alguna no es favorable, se busca otra alternativa mejor y factible.

\section{Responsable de programa, estructura y presupuesto}

El modelo SSeTGIS como promotor de un cambio de cultura de gestión y de trabajo de todos los miembros de la organización requiere de las políticas, un programa de implementación y otro permanente de mejora continua. De acuerdo al marco jurídico, dependiendo del número de personal, las organizaciones deben contar con:

- Un Responsable de SSeTMAPC competente para coordinar, capacitar y asesorar a la organización en las propias materias 
SSeTMAPC, así como de documentarlo. Puede ser contratado, asignado para hacer las funciones o un consultor. Existe la posibilidad de ser aplicado por el dirigente de las micro, pequeñas y medianas empresas, formándose y asesorándose de consultores en áreas específicas para cumplir la normatividad, obtener los máximos beneficios y la sustentabilidad. En empresas grandes ${ }^{23}$, dicho responsable deberá ser de alto nivel, de preferencia inmediato al director, pues mostraría el compromiso con la filosofía expresada en las políticas.

- Una estructura organizacional técnica es necesaria para organizaciones grandes, compuesta por especialistas en cada área disciplinar SSeTMAPC que se gestiona y un responsable por cada unidad de proceso. ${ }^{24}$

- Un presupuesto que les permita implementar y mantener los programas para dar cumplimiento al marco jurídico que en esas materias aplica a la organización. No se trata de trabajar más, sino diferente. Tampoco se trata de agregar gasto, sino de asegurar que el que se tiene se aplique mejor pasándolo por ese mismo filtro de la "pausa SSeTGIS" establecido como política, aportando a la sustentabilidad económica, ambiental y social al evitar el uso posterior de recursos para corregir, reparar o vigilar procesos inadecuados y sus consecuencias.

\section{El proceso}

El modelo SSeTGIS, y su aplicación, es un proceso de ciclos anuales. Cada ciclo tiene tres fases, que se cubren en siete pasos; el octavo paso es el inicio del ciclo subsecuente; se muestra en la Figura $1 .^{25}$ El ciclo se cierra con una evaluación, la misma que se convierte en el punto de partida del siguiente ciclo.

\section{Figura 1.}

Proceso del modelo SSeTGIS de ciclos de mejora continua.

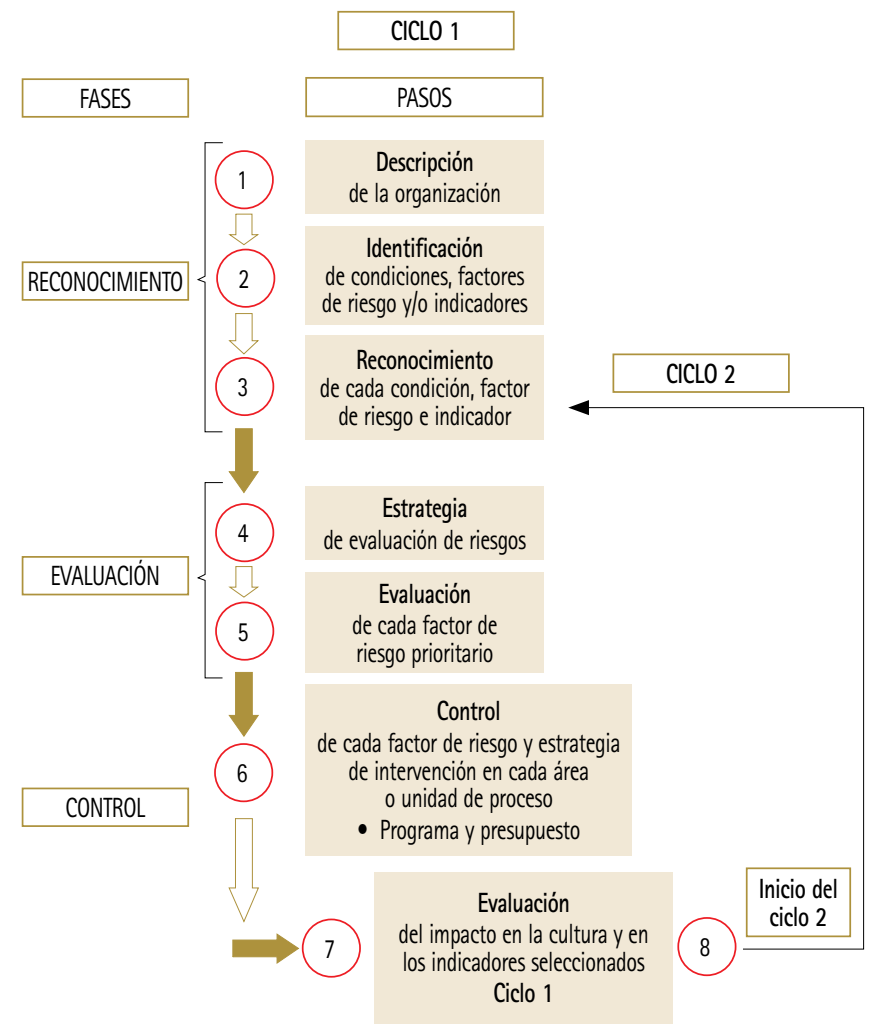

\section{- La gestión. El inicio}

El proceso inicia en el nivel organizacional con la gestión ante las partes interesadas, tanto el responsable de la organización como los trabajadores o sus representantes, y con el responsable de la estructura organizacional de SSeTMAPC, si cuenta con una. Se presenta la propuesta que muestre el modelo, los beneficios y las oportunidades ante las debilidades y amenazas en un documento conciso. Este es un paso desafiante y clave.

Para ello es necesario conocer la organización y su cultura; además: el análisis histórico de emergencias; su ubicación geográfica, para identificar vulnerabilidad para el entorno y desde él; la descripción general del proceso productivo del bien o servicio, las unidades de proceso, su programa operativo y su presupuesto; la estructura organizacional, los diferentes puestos de trabajo, el personal que los ocupa, las funciones y tareas que realizan, dónde y con qué, e identificar sus estrategias de comunicación.

\section{- El proyecto}

Una vez que se conoce la organización, se continúa con la estructuración del proyecto y el programa operativo por parte del promotor o el responsable de la estructura organizacional de SSeTMAPC, de acuerdo a las necesidades específicas y expresas de la organización. Incluso adaptándolo al ejercicio en desarrollo o de cara a un nuevo periodo presupuestal, planteando de acuerdo con el compromiso de la organización, los objetivos, metas e indicadores.

Es importante una campaña de comunicación dirigida al personal, para la sensibilización sobre las políticas, el programa y su propósito; después otra de socialización de las diferentes actividades, su propósito y sus resultados.

- Ciclo de implementación del modelo SSeTGIS

El proceso en su ciclo inicial se desarrolla como un programa con las acciones de cada paso, de acuerdo a los objetivos para cada uno de los componentes de SSeTMAPC normados en el marco jurídico, en cada nivel de actuación, individual, lugar de trabajo y organizacional. Para lo cual se aplica la técnica más adecuada ya sea análisis documental de informes y notas diversas, entrevista al personal, observación no participante, y métodos de evaluación estandarizados o estimación por método numérico.

Para organizaciones grandes, medianas y, en algunos casos, pequeñas, deberán establecerse las acciones para cada área física, sección o unidad de proceso en que esté dividida y consensuarse tanto con el responsable de dicha área o unidad como con su personal, quienes las realizarán contando en todo momento con la asesoria, capacitación y coordinación del responsable o la estructura de SSeTMAPC.

El proceso se desarrolla según los ocho pasos indicados en la Figura 1, como sigue:

\section{Análisis de información de la organización}

Es necesario revisar y actualizar la información recabada en el inicio y complementar con las necesidades de la organización percibidas por la dirección, los trabajadores o sus representantes.

\section{Identificación de las condiciones, los factores de riesgo, e} indicadores

2.1. Elaborar un listado de identificación con las condiciones, los factores de riesgo e indicadores de cada área SSeTMAPC, asimismo los indicadores de calidad y productividad que la orga- 
nización valora para una gestión integral. Sirve utilizar un formato que contenga: condición, factor de riesgo o indicador; normatividad de SSeTMAPC correspondiente; espacio para indicar la aplicabilidad; y área física o unidad de proceso donde se encuentra, si fuera el caso.

\section{Reconocimiento en el lugar de trabajo, de cada condición, factor de riesgo o indicador que aplique de la normatividad de SSeTMAPC}

3.1. Establecer el objetivo y alcance del estudio-reconocimiento para la implementación o el mantenimiento del modelo SSeTGIS. Considerar personal, puestos de trabajo, áreas y factores prioritarios de acuerdo a las necesidades y los recursos disponibles. En un primer ciclo, se pueden considerar los requerimientos normativos de prioridad uno como objetivo, en los subsecuentes cubrir los de prioridad dos y así sucesivamente.

3.2. Obtener un listado de verificación para cada condición, factor de riesgo o indicador, ya sea por cada normativa de salud, seguridad e higiene en el trabajo, medio ambiente o protección civil; o bien, uno que contenga los requerimientos de todas ellas. Sirve utilizar un formato para cada condición, factor de riesgo o indicador que contenga: requerimientos normativos a verificar; espacio para indicar aplicabilidad; otro para indicar el cumplimiento en caso de aplicar; área física donde se encuentra; fuente generadora; y observaciones o recomendaciones. Para el cumplimiento también se puede utilizar una escala por niveles refiriendo los criterios empleados. Una alternativa más completa son las llamadas matrices de identificación de peligros y evaluación de riesgos (IPER). ${ }^{26}$

3.3. Realizar un recorrido sensorial por el lugar de trabajo observando y registrando en el listado de verificación el estado de las condiciones y factores de riesgo.

3.4. Obtener los inventarios, los planos, el mapa de riesgos y elaborar un cuadro de hallazgos. Los inventarios son de personal, de sustancias químicas, de fuentes generadoras y emisión de contaminantes, de maquinaria y equipo, de instalaciones peligrosas y de riesgos por fenómenos perturbadores de origen natural.

3.5. Realizar el análisis de peligrosidad para cada factor de riesgo mediante metodología normada o reconocida para priorizar las necesidades de evaluación y de control.

3.6. Registrar el tiempo y otros recursos invertidos que sirvan de referencia para planeaciones futuras.

3.7. Revisar registros o informes de los exámenes médicos realizados, ya sean de ingreso, periódicos o específicos; las estadísticas de los riesgos de trabajo, tanto accidentes de trabajo como enfermedades profesionales y casos de enfermedad general; y las campañas de promoción de la salud.

3.8. Elaborar un informe del reconocimiento como diagnóstico situacional de SSeTMAPC, con la descripción de la organización, el nivel de cumplimiento de la normatividad, el estado de los indicadores de calidad y de productividad y las recomendaciones generales y específicas. Anexar las evidencias correspondientes.

\section{Elaboración de la estrategia de evaluación de riesgos}

4.1. Priorizar los factores de riesgo a partir del análisis de peligrosidad del paso 3.

4.2. Capturar en un formato que guie el registro de los datos de cada factor de riesgo y de la exposición de las personas para la asignación de la prioridad, así como la metodología para su evalua- ción posterior. Se sugiere registrar: unidad de proceso, área física, puesto de trabajo, número de trabajadores en el puesto, tareas principales del puesto, riesgo (por condición, factor o fenómeno perturbador), fuente generadora o emisora, metodología para medirlo o estimarlo, instrumento, muestra (número de trabajadores $u$ otras personas), prioridad (justificada), controles existentes y propuestos en el diagnóstico.

\section{Evaluación de los factores de riesgo}

5.1. Medir los factores de riesgos con método e instrumentos normados.

5.2. Estimar por método numérico reconocido los riesgos que no tienen una medición directa.

5.3. Analizar e interpretar los resultados de la medición y compararlos con los niveles aceptables y normados como evaluación del riesgo para recomendar medidas de control.

5.4. Determinar la prioridad de las medidas recomendadas bajo los criterios de: efectos a la salud del personal ocupacionalmente expuesto, riesgo para las instalaciones y el ambiente. Preferir controles colectivos en la fuente generadora o emisora del factor de riesgo, luego en el ambiente de trabajo y como tercera elección las medidas en la persona. Es conveniente establecer el impacto que estas medidas tendrían en indicadores de calidad y productividad, mostrando el efecto sinérgico de la acción y con el compromiso de evaluarlo posteriormente.

5.5. Elaborar el informe de la evaluación que incluya la estrategia de evaluación de riesgo priorizada, los resultados de la evaluación y las medidas recomendadas para el control de riesgos.

\section{Elaboración del programa SSeTGIS de control de riesgos y su presupuesto}

6.1. Elaborar el programa con las acciones, ya sea por recomendaciones técnicas, por método participativo o ambos, integrando grupos que hayan trabajado a través de un proceso de investigación-acción como un procedimiento que aporta a una cultura de salud y seguridad con calidad y productividad, y alcanza las máximas ventajas. El periodo del programa normalmente es anual, como el presupuesto.

6.2. Incluir en el programa los datos de la organización o área y para cada componente del modelo SSeTGIS: la acción, su cita en la normatividad y en los objetivos de la organización, persona o puesto responsable, horas-persona estimadas, monto económico y cronograma anual (o fecha de término como mínimo).

6.3. Es importante considerar en el programa medidas inmediatas, de bajo costo, así como eventos y acciones diversas para comunicar, motivar y mantener el interés en la SSeTMAPC: proponer y participar en la coordinación de juntas informativas para comunicar resultados; señalización, carteles, spots, tripticos, campañas, concursos; paneles de discusión, conferencias, pláticas, exposiciones, jornadas sobre los diferentes temas, entre otros. Considerar medios electrónicos e impresos.

6.4. Para hacer operativa la visión del modelo persona por persona, se puede plantear una "pausa SSeTGIS" para que cada miembro de la organización valore su decisión y acción de acuerdo con los efectos a la SSeTMAPC, a la calidad o a la productividad.

6.5. Elaboración del presupuesto general SSeTGIS y gestionar el específico por unidad de proceso necesario para realizar las acciones del programa, correspondientes a sus actividades, instalaciones y su personal. Identificar para cada acción, en cada componente, el monto requerido y la fecha o periodo de 
aplicación. Acompañar con las cotizaciones de tantas partidas como sea posible.

6.6. Ejecución del programa SSeTGIS en tiempo y forma.

\section{Evaluación del ciclo completo}

Se requiere evaluar cada ciclo anualmente, en función del cumplimiento de los objetivos planteados y los resultados del programa, participativamente es mejor. Elaborar el informe indicando las tareas pendientes y las recomendadas para cada una de las fases del siguiente ciclo del proceso de mejora continua.

\section{Inicio de un nuevo ciclo}

El punto de partida del proceso de mejora continua es la evaluación del ciclo anterior que permita contar con un diagnóstico situacional actualizado de SSeTMAPC y de los indicadores de calidad y productividad, estableciendo las necesidades, los recursos, los objetivos y la nueva estrategia de evaluación; posteriormente, el programa de evaluación y de control para el siguiente periodo anual.

Es importante indicar que, iniciado el proceso, requiere de una fase de anticipación, la cual consiste en acciones dirigidas a identificar, estimar y modificar desde la evaluación de proyectos, los riesgos potenciales. Los proyectos pueden ser recursos innovadores físicos o humanos para el desarrollo de la organización. Es conveniente establecer un procedimiento para proyectos antes de su ejecución, el cual contemple el análisis de riesgos para la SSeTMAPC de manera que se pueda seleccionar la alternativa de menor riesgo y exposición de acuerdo con el tipo de organización. ${ }^{27}$

\section{METODOLOGÍA POR COMPONENTE}

La diversidad de factores involucrados en las organizaciones, componentes de cada área gestionada en el modelo SSeTGIS y el programa derivado de él, requieren de una metodología, técnicas e instrumentos específicos. Se trata de métodos estandarizados, muchos de ellos son normas obligatorias dentro del marco jurídico de cada país (véase resumen en la Tabla 1).

Un sistema de información es necesario para recolectar, analizar y reportar regularmente datos para la toma de decisiones y el cumplimiento legal en cada materia.

Los tres pasos del reconocimiento determinan la prioridad de los puestos, tareas, áreas y factores de riesgo para la siguiente fase de evaluación mediante las técnicas cualitativas de análisis documental, observación no participante y entrevista.

La evaluación consiste en la cuantificación de cada componente prioritario, sea condición, factor de riesgo o indicador en cada unidad de proceso, así como la exposición de las personas a él. Se realiza a través de la medición directa o por método numérico válido y

Tabla 1. Metodología del modelo SSeTGIS por área para cada componente, etapa de un ciclo y nivel de actuación.

\begin{tabular}{|c|c|c|c|c|c|}
\hline $\begin{array}{l}\text { Área del } \\
\text { modelo } \\
\text { SSeTGIS }\end{array}$ & $\begin{array}{l}\text { Componentes } \\
\text { (condiciones, factores de riesgo e indicadores) }\end{array}$ & Técnica/ instrumentos & Técnica/ instrumentos & $\begin{array}{l}\text { Control de riesgos / } \\
\text { intervención } \\
\text { organizacional } \\
\text { Técnica/ instrumentos }\end{array}$ & $\begin{array}{l}\text { Nivel } \\
\text { de actuación }\end{array}$ \\
\hline \multirow[t]{2}{*}{ Salud } & $\begin{array}{l}\text { Salud integral de cada trabajador(a) médico, nutricional, } \\
\text { laboratorial, psicosocial y de antecedentes laborales. }\end{array}$ & $\begin{array}{l}\text { Indicados } \\
\text { Método std }\end{array}$ & Especificos & \multirow{2}{*}{$\begin{array}{c}\text { Multidisciplinario } \\
\text { Tratamiento y } \\
\text { recomendaciones para } \\
\text { trabajador(a) y la organización. }\end{array}$} & \multirow[t]{2}{*}{$\begin{array}{l}\text { Individual } \\
\text { Trabajador(a) }\end{array}$} \\
\hline & $\begin{array}{l}\text { Exámenes médicos de ingreso y periódicos, } \\
\text { registro de riesgos de trabajo. }\end{array}$ & $\begin{array}{l}\text { Método std } \\
\text { normatividad }\end{array}$ & $\begin{array}{l}\text { Método std } \\
\text { normatividad }\end{array}$ & & \\
\hline \multirow{6}{*}{$\begin{array}{l}\text { Seguridad } \\
\text { e higiene en } \\
\text { el trabajo }\end{array}$} & Biológicos & \multirow{6}{*}{$\begin{array}{l}\text { Método std } \\
\text { normatividad } \\
\text { y participativo }\end{array}$} & \multirow{6}{*}{$\begin{array}{l}\text { Método std } \\
\text { normatividad }\end{array}$} & \multirow{6}{*}{$\begin{array}{l}\text { Mejores prácticas / } \\
\text { participativo para reducir o } \\
\text { eliminar los factores de riesgo } \\
\text { y mejorar los métodos de } \\
\text { trabajo por unidad de proceso } \\
\text { y área desde cada } \\
\text { responsable y cada } \\
\text { trabajador(a). } \\
\text { Establecer a partir del } \\
\text { reconocimiento y evaluación. }\end{array}$} & \multirow{7}{*}{$\begin{array}{l}\text { Lugar de } \\
\text { trabajo }\end{array}$} \\
\hline & Químicos & & & & \\
\hline & $\begin{array}{l}\text { Físicos (ruido, condiciones térmicas, iluminación, } \\
\text { radiaciones, vibraciones). }\end{array}$ & & & & \\
\hline & $\begin{array}{l}\text { Ergonómicos (Postura, fuerza, repetitividad, } \\
\text { duración, ritmo, carga mental). }\end{array}$ & & & & \\
\hline & $\begin{array}{l}\text { Psicosociales del trabajo (contexto de trabajo } \\
\text { y contenido de trabajo). }\end{array}$ & & & & \\
\hline & $\begin{array}{l}\text { Mecánico-eléctricos (maquinaria e instalaciones), } \\
\text { estructurales (edificios) y análisis de riesgos de } \\
\text { instalaciones peligrosas (uso de sustancias } \\
\text { químicas) e incendios. }\end{array}$ & & & & \\
\hline $\begin{array}{l}\text { Medio } \\
\text { ambiente }\end{array}$ & $\begin{array}{l}\text { Emisiones al aire, agua y suelo. } \\
\text { Consumo de energía y aprovechamiento de agua. }\end{array}$ & $\begin{array}{l}\text { Método std } \\
\text { normatividad }\end{array}$ & $\begin{array}{l}\text { Método std } \\
\text { normatividad }\end{array}$ & $\begin{array}{l}\text { Mejores prácticas / } \\
\text { participativo }\end{array}$ & \\
\hline $\begin{array}{l}\text { Protección } \\
\text { civil }\end{array}$ & $\begin{array}{l}\text { Acciones preventivas en sitios de riesgo por fenómenos } \\
\text { perturbadores (naturales y antrópicos), brigadistas y } \\
\text { personas capacitados, y emergencias atendidas. }\end{array}$ & Normatividad & Especificos & $\begin{array}{l}\text { Mejores prácticas / } \\
\text { participativo }\end{array}$ & $\begin{array}{l}\text { Contexto del } \\
\text { lugar de } \\
\text { trabajo }\end{array}$ \\
\hline \multirow{5}{*}{$\begin{array}{l}\text { Gestión } \\
\text { integral }\end{array}$} & Cultura (Misión, visión y valores). & Cuest. std & Cuest. std & & \multirow{7}{*}{$\begin{array}{c}\text { Organizacional } \\
\text { Gestión del } \\
\text { talento } \\
\text { humano y } \\
\text { planeación } \\
\text { estratégica. }\end{array}$} \\
\hline & Liderazgo y compromiso (políticas en áreas SSeTGIS). & & & & \\
\hline & Planeación estratégica (objetivos, metas). & Método std & Método std & Establecer a partir del & \\
\hline & Estructura organizacional de áreas SSeTGIS. & Auditorias & Auditorias & reconocimiento y evaluación. & \\
\hline & $\begin{array}{l}\text { Sistema de gestión, información, capacitación } \\
\text { y comunicación. }\end{array}$ & Participativo & Participativo & & \\
\hline Calidad & Indicadores seleccionados. & \multirow[t]{2}{*}{ Específicos } & \multirow[t]{2}{*}{ Especificos } & \multirow{2}{*}{$\begin{array}{l}\text { Establecer a partir del } \\
\text { reconocimiento y evaluación. }\end{array}$} & \\
\hline Productividad & Indicadores seleccionados. & & & & \\
\hline
\end{tabular}

Fuente: Elaboración propia. SSeTGIS: Salud y seguridad en el trabajo con gestión integral para la sustentabilidad. Método std: Método estandarizado.

Normatividad: del marco jurídico nacional de cada pais o de lineamientos internacionales. Cuest. std: Cuestionario estandarizado. 
confiable, para posteriormente compararlo con un valor de referencia y establecer la acción de control necesaria. La técnica e instrumento corresponden a la naturaleza del componente: químico, físico, biológico, ergonómico, psicosocial, mecánico, eléctrico, estructural o natural.

La fase de control no se limita a una metodología particular, sino que se buscan alternativas disponibles en el mercado para lograr dicho control. De acuerdo con la normatividad internacional, debe primar el control colectivo sobre el individual, lo que implica ir al origen de los riesgos en los niveles organizacional y del lugar de trabajo, aprovechando la sinergia, antes que persona por persona. La norma OHSAS 18001 establece la jerarquía: “a) eliminación; b) sustitución; c) controles de ingeniería; d) señalización/advertencia y/o controles administrativos; y, e) equipo de protección personal".28

Siempre es recomendable la participación de los trabajadores y trabajadoras integrados en equipos de análisis y resolución de problemas en alguna de las fases o en todas, como en la investigación-acción. Deben asegurarse recursos para implementar las medidas acordadas. Los factores psicosociales son en los que se evalúa la percepción de los trabajadores sobre los componentes de los niveles individual, lugar de trabajo y organizacional del modelo, con un instrumento estandarizado multidimensional válido y confiable.

\section{NIVELES DE ACTUACIÓN}

El modelo tiene tres niveles de actuación:

- En el nivel individual, se considera tanto la promoción de la salud como su evaluación integral -biopsicosocial-, el tratamiento y recomendaciones hacia el trabajador para mantener o mejorar salud, y hacia la organización en relación a los factores del lugar de trabajo y de la organización del trabajo que pueden estar afectando al personal. Se requiere la intervención de un equipo multidisciplinario de la salud: medicina, nutrición, enfermería, psicología así como de un profesional de seguridad y salud en el trabajo. Se promueven y ofrecen al personal herramientas para estilos de

\section{Figura 2.}

Nivel individual del modelo SSeTGIS. Evaluación de salud integral.

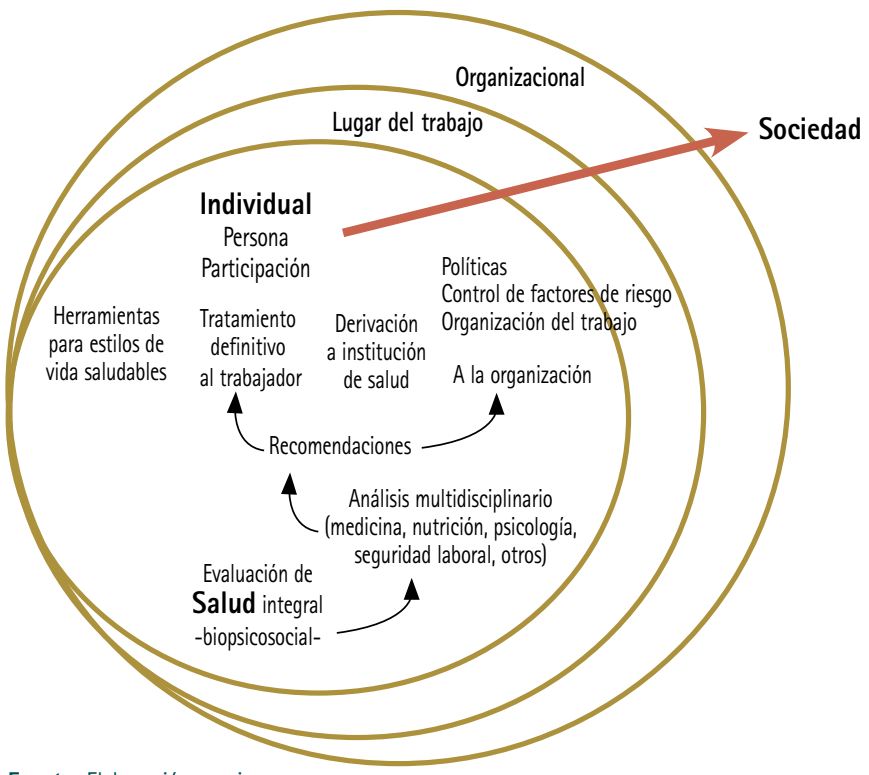

Fuente: Elaboración propia.
Figura 3.

Nivel del lugar de trabajo del modelo SSeTGIS y sus componentes.

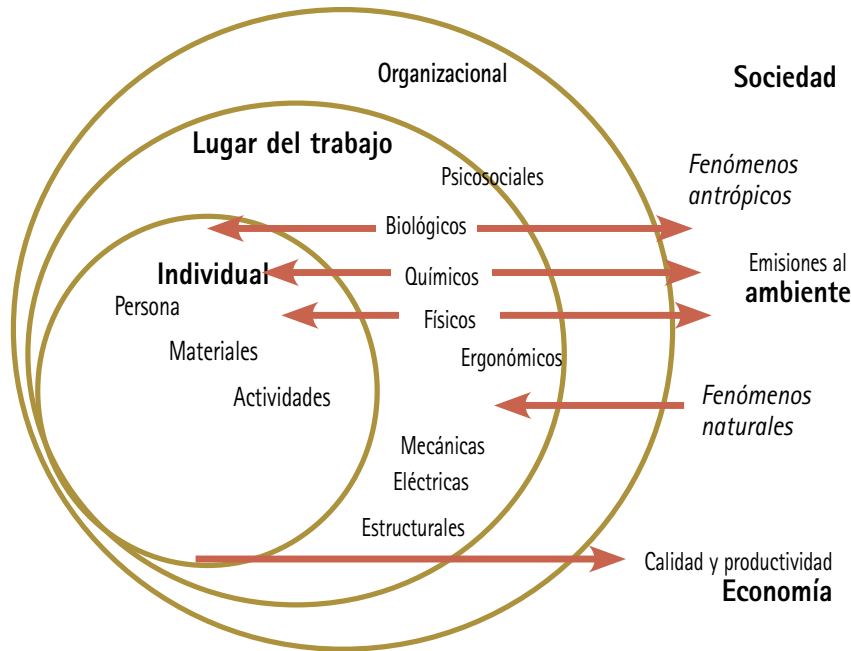

Fuente: Elaboración propia

vida saludables, una mayor satisfacción en el empleo y sus resultados, favoreciendo la salud y la participación, ambas necesidades humanas (véase Figura 2).

- En el nivel del lugar de trabajo, los trabajadores y trabajadoras realizan sus actividades laborales con diversos materiales expuestos a factores de riesgo y en determinadas condiciones. En este nivel se generan riesgos potenciales a la salud y la seguridad, pero también para la calidad, la productividad y el medio ambiente, pudiéndose convertir en riesgos antrópicos (químico-tecnológicos, sanitario-ecológicos y socio-organizativos) para el entorno. Éste, a su vez, presenta riesgos por fenómenos perturbadores naturales (geológicos e hidrometeorológicos) para las instalaciones y el personal que deben prevenirse como protección civil (véase Figura 3).

Desde la integralidad que el modelo SSeTGIS propone, es en ese mismo lugar donde se inicia el proceso de mejora y puede medirse.

- El nivel organizacional es donde inicia y termina el proceso propuesto por el modelo SSeTGIS al centrarse en las personas como gestión del talento humano que reconoce su contribución a la organización en la resolución de problemas mediante la participación, trabajo decente y la responsabilidad social para la sustentabilidad, estableciendo que la SSeTMAPC está al nivel de la calidad y productividad en sus políticas y lineamientos. Se apoya en el responsable de SSeTMAPC y considera a los representantes de los trabajadores.

Implica la comunicación, información y formación de todos y cada uno de los miembros de la organización en una cultura de trabajo y de gestión integral para que en sus análisis, decisiones y acciones consideren la SSeTMAPC con calidad y productividad, según las políticas y los objetivos establecidos en la planeación estratégica. Promueve la óptima organización del trabajo en el proceso productivo del bien o servicio, clarificando para el personal su rol, su puesto, las funciones y tareas, así como suministrando los recursos necesarios (véase Figura 4).

Lo anterior considera el análisis de los puestos de trabajo actuales y su posible rediseño a favor de la SSeTMAPC con calidad y productividad; el desarrollo personal, el reclutamiento, selección y gestión del talento humano a incorporar; así como la comunicación para sensibilizar en las políticas y la socialización de la 
Figura 4.

Nivel organizacional.

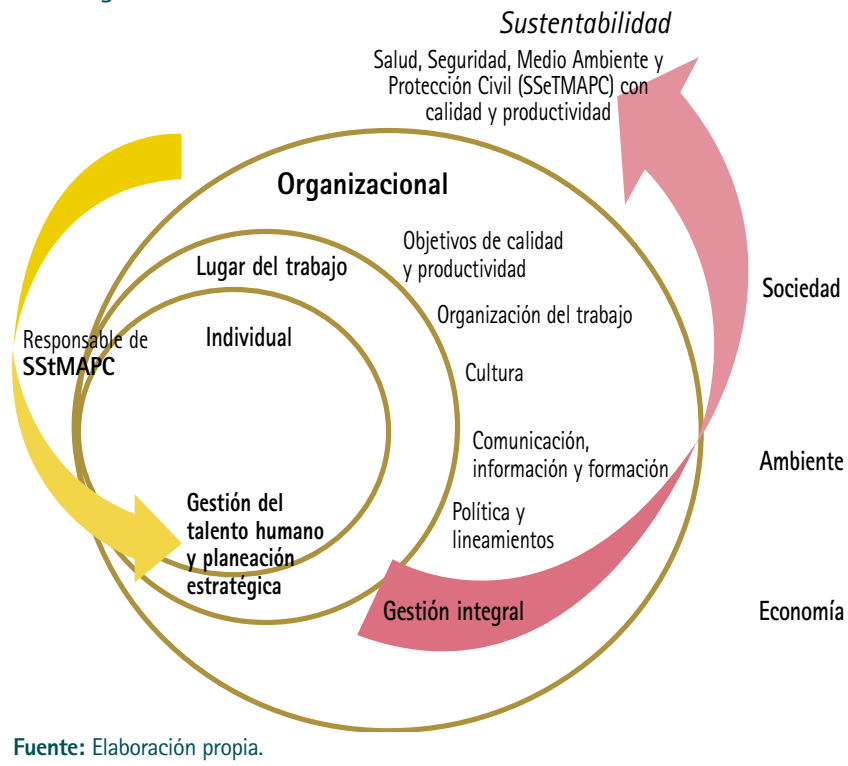

visión según el modelo SSeTGIS, del programa y sus resultados en cuanto al cumplimiento de las metas y el logro de los objetivos, misión y visión de la organización. Contempla, según la normatividad, la evaluación de los factores psicosociales para su control con una intervención pertinente y oportuna.

La gestión integral logra sinergia de recursos a favor de todas las áreas.

La representación de los ciclos de mejora continua en espiral, de tres fases, puede verse en la Figura 5 y el modelo completo en la Figura 6.

Figura 5.

Ciclos de mejora continua de reconocer, evaluar y controlar-intervenir.

\section{Sustentabilidad}

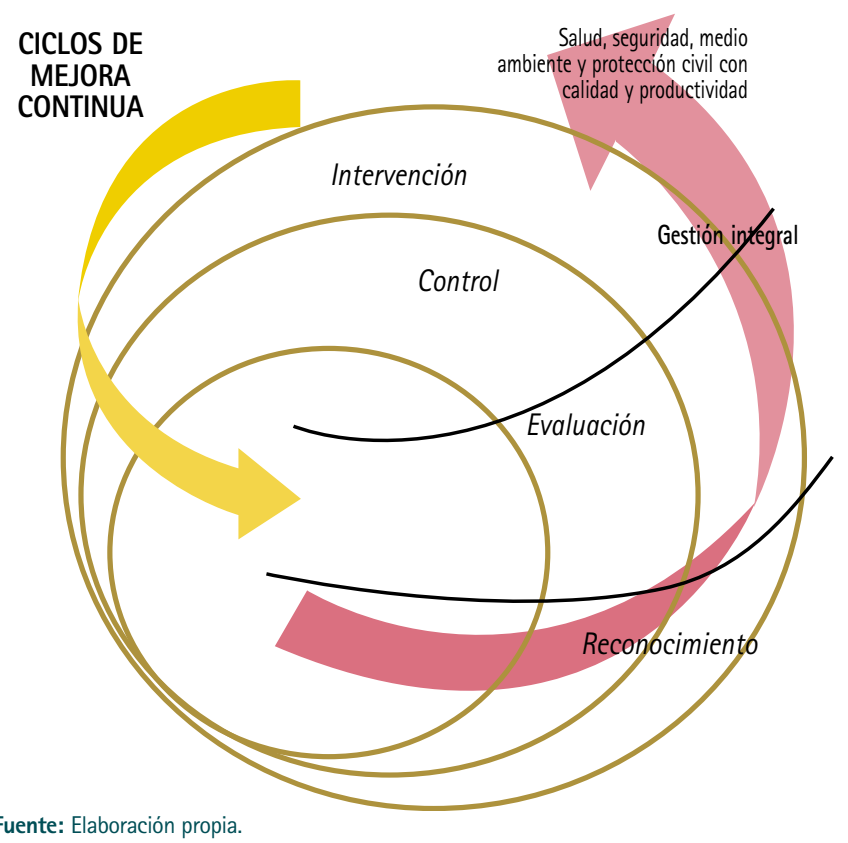

NOTAS FINALES

Se han realizado ejercicios de aplicación del modelo en una decena de organizaciones de todo tamaño y de los tres sectores económicos en los cuales se efectuó el reconocimiento de los componentes de SSeTMAPC, la evaluación de los prioritarios, se propusieron medidas logrando la implementación de algunas que mostraron su efectividad. Sin embargo, el reto sigue siendo la acción congruente con los propósitos de sustentabilidad desde la dirección y en cada persona.

Los procesos participativos han sido excelentes para establecer planes de acción pertinentes; sin embargo, su realización es un desafío que se sigue estudiando. La alternativa de una intervención organizacional por expertos que desarrollen un programa adecuado de corto plazo y un acompañamiento posterior para el logro de los objetivos y los propósitos que el modelo promueve, es una inversión que debe presupuestarse.

Se desarrolló un instrumento cuantitativo en hoja de cálculo para el reconocimiento integral de SSeTMAPC basado en la normatividad que facilite medir su cumplimiento como diagnóstico y luego la mejora conforme se asigna recursos, así como diversos formatos para la recolección de datos. Son aplicables tanto para instalaciones existentes como para el estudio de factibilidad de proyectos de inversión.

\section{CONCLUSIÓN}

Es importante que las organizaciones tengan alternativas de modelos integrales que les permitan lograr sus objetivos y cumplir su misión de la mejor manera posible, maximizando beneficios y disminuyendo daños y costos. ${ }^{1,5}$ Establecerse como un proceso que involucre a toda la organización a partir de los líderes, mediante sus políticas y lineamientos, como lo señala la ISO29 y OIT.30 También, contar con un responsable de la coordinación y asesor en las áreas del modelo, y un presupuesto.

El modelo SSeTGIS es de gestión integral, holístico desde la perspectiva de la salud y seguridad en el trabajo sin limitarse a ellas. Integra el cuidado del medio ambiente y la protección civil con calidad y productividad para la sustentabilidad de las organizaciones, aprovechando la sinergia en el análisis, la decisión y la acción de cada trabajador así como de los recursos invertidos.

Existe la posibilidad de ser aplicado por el dirigente de las micro y pequeñas empresas formándose y asesorándose de consultores en áreas específicas SSeTMAPC que necesite, para obtener los máximos beneficios, cumplir la normatividad y alcanzar la sustentabilidad con gestión integral de su organización.

El modelo SSeTGIS comparte la fundamentación, filosofía y perspectiva de la teoría de sistemas ${ }^{13}$ de otros, como el de Responsabilidad social $^{6}$, el de Entornos laborales saludables ${ }^{7}$ y el de Organizaciones saludables y resilientes ${ }^{14}$, de ser holísticos, multidisciplinares, participativos y de intervención psicosocial. ${ }^{31}$ No se identificó algún modelo similar en el contexto latinoamericano, pero sí los argumentos para contar con uno. ${ }^{9,11-12}$ Lo diferente del modelo SSeTGIS está en tomar la salud y seguridad en el trabajo como perspectiva centrada en las personas como primeras beneficiarias y partícipes en la cultura laboral que promueve; ubica en el lugar de trabajo inmediato, el personal y sus actividades laborales el origen de los riesgos potenciales, pero también de la mejora continua como proceso; y promueve la aplicación de metodología adecuada para la medición de los avances en todas las áreas para verificar la sinergia.

Toma como marco los convenios internacionales y las legislaciones 
Figura 6. Modelo SSeTGIS. Componentes por niveles de actuación y proceso.

\section{CICLOS DE MEJORA CONTINUA}

\section{Sustentabilidad}

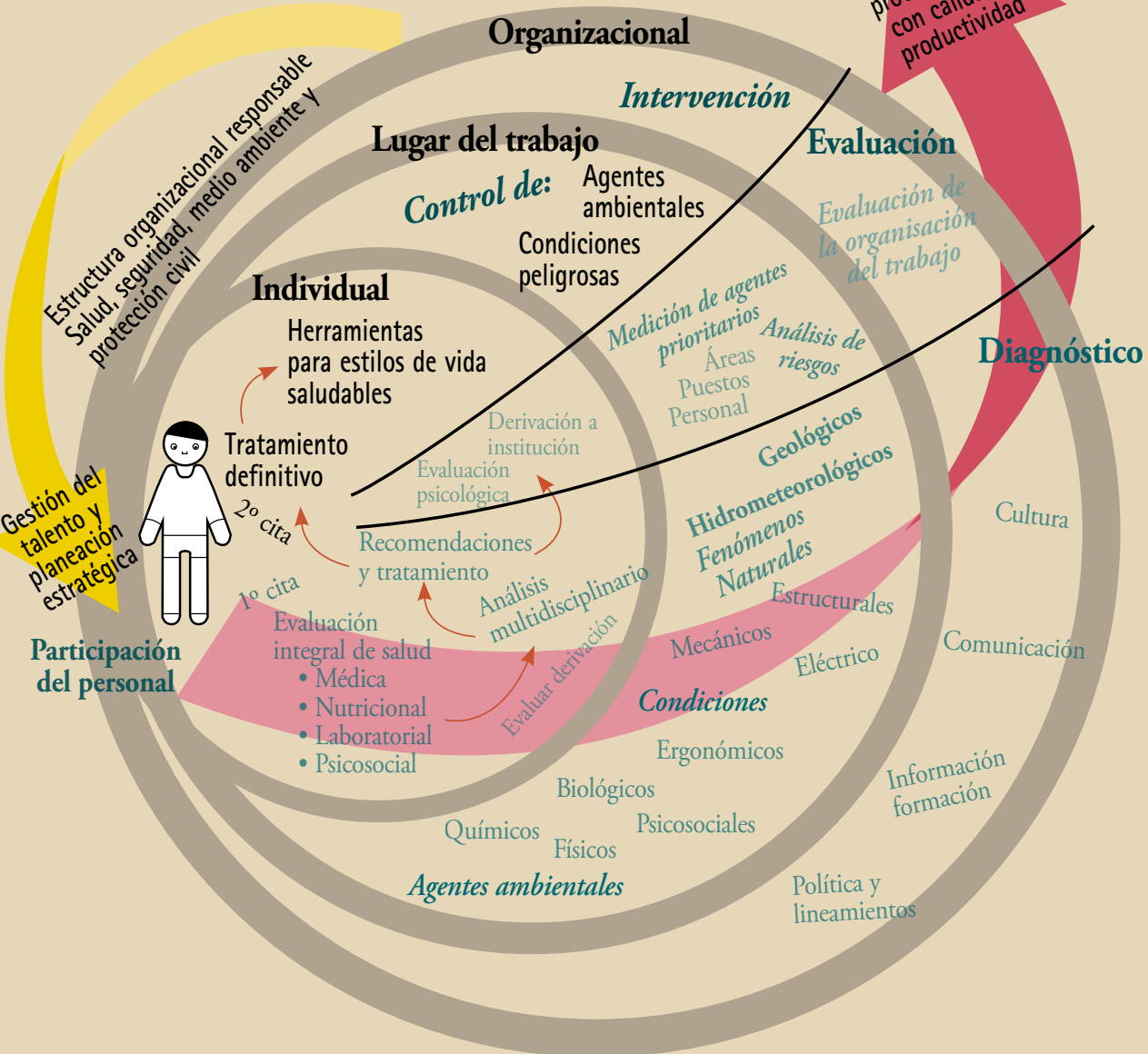

\section{Sociedad}

\section{Ambiente}

\section{Economía}

Fuente: Elaboración propia.

nacionales que establecen requerimientos mínimos en materia de SSeTMAPC, y de los indicadores de calidad y productividad. Propone una "pausa" durante la cual cada persona valore previamente sus acciones por los efectos a la SSeTMAPC, la calidad y la productividad que tendrán, a manera de filtro para determinar si las realiza o busca otra alternativa.

En los ejercicios de aplicación del modelo, las fases del ciclo de reconocimiento de los componentes de SSeTMAPC y de evaluación de los prioritarios, se desarrollan sin mayores dificultades por personal capacitado; el reto está en la fase de control, la implementación de las medidas en los tres niveles de actuación, pero en particular en el organizacional, lo cual se sigue estudiando. Alternativas para atender el desafío son intervención organizacional por expertos y la participación de los trabajadores en la investigación-acción como estrategia para el proceso transformador hacia la sustentabilidad.

\section{Siglario}

AENOR Asociación Española de Normalización

IPER Identificación de Peligros y Evaluación de Riesgos

ISO

International Standard Organization
OIT Organización Internacional del Trabajo

OMS Organización Mundial de la Salud

SHT Seguridad e Higiene en el Trabajo

SSeT Salud y Seguridad en el Trabajo

SSeTGIS Salud y Seguridad en el Trabajo con Gestión Integral para la Sustentabilidad de las organizaciones (léase "setgis")

SSeTMAPC Salud y Seguridad en el Trabajo, Medio Ambiente y Protección Civil

\section{Agradecimiento}

Son muchos las personas participantes en el desarrollo, aplicación y evaluación del modelo SSeTGIS. Mi agradecimiento a:

Los trabajadores y directivos de las organizaciones por su confianza. Los estudiantes y egresados de la Licenciatura en Seguridad laboral, protección civil y emergencias por su esfuerzo, determinación, aportaciones técnicas e instrumentales y de sistematización.

Los profesionales e investigadores de diversas disciplinas, quienes han contribuido al desarrollo, implementación y evaluación del proceso y sus resultados. 
1. Oficina Internacional del Trabajo. Investigación de accidentes del trabajo y enfermedades profesionales [en línea]. Ginebra: OIT; 2015 [consultado 15 dic 2016]. Disponible en: http://www.ilo.org/wcmsp5/groups/public/@ed_dialogue/ @lab_admin/documents/publication/wcms_346717.pdf

2. Chu C. De una promoción de la salud en el lugar de trabajo hacia la gestión integradora de la salud en el lugar de trabajo: Tendencias y evolución. GOHNET [en línea]. 2003 [consultado 15 ene 2016]; (6):1-4. Disponible en: http://www. who.int/occupational_health/publications/newsletter/en/gohnet6s.pdf?ua=1

3. Organización de Naciones Unidas (ONU). Objetivos de desarrollo sostenible [en linea]. Ginebra: ONU; 2016 [consultado 15 dic 2016]. Disponible en: http://www. undp.org/content/undp/en/home/sustainable-development-goals.html

4. Max-Neef M, Elizalde A, Hopenhayn M. Desarrollo a escala humana: opciones para el futuro [en línea]. Santiago de Chile: Ciudades para un Futuro más Sostenible; 2010 [consultado 15 dic 2016]. Disponible en: http://habitat.aq.upm. es/deh/adeh.pdf

5. Oficina de las Naciones Unidas para la Reducción del Riesgo de Desastres (UNISDR). Hacia el desarrollo sostenible: El futuro de la gestión del riesgo de desastres. Informe de evaluación global sobre la reducción del riesgo de desastres [en línea]. Ginebra: UNISDR; 2015 [consultado 15 dic 2016]. Disponible en: http:// www.preventionweb.net/english/hyogo/gar/2015/en/gar-pdf/GAR2015_SP.pdf

6. International Standard Organization (ISO). Norma ISO 26000:2010. Guía de responsabilidad social empresarial [en línea]. Ginebra: ISO; 2010 [consultado el 15 de diciembre de 2016]. Disponible en: http://fundahrse.org/wp-content/ uploads/2016/01/Borrador-ISO-26000.pdf

7. Organización Mundial de la Salud (OMS). Entornos laborales saludables: Fundamentos y modelo de la OMS [en línea] Ginebra: OMS; 2010 [consultado 10 jun 2016]. Disponible en: http://www.who.int

8. Salanova M, Martínez IM, Llorens S. Una mirada más "positiva" a la salud ocupacional desde la psicología organizacional positiva en tiempos de crisis: Aportaciones desde el equipo de investigación WoNT. Pap Psicól. 2014; 35(1):22-30.

9. Neira M. Healthy workplaces: a model for action. For employers, workers, policymakers and practitioners [en línea] Ginebra: Organización Mundial de la Salud. 2010 [consultado 15 dic 2016]. Disponible en: http://www.who.int/occupational_health/publications/healthy_workplaces_model.pdf

10. Anaya A. Condiciones de seguridad e higiene del trabajo en organizaciones. En: Espinosa $J A$, editor. Municipio modelo de desarrollo turistico sustentable. Diagnóstico integral de Gómez Farias, Jalisco. Guadalajara: Universidad de Guadalajara-Centro Universitario del Sur; 2007. p. 75-92.

11. Raffo E, Raez L, Cachay 0. Riesgos psicosociales. Industrial Data. 2013; 16(1):70-79.

12. Chiavenato I. Administración de recursos humanos, el capital humano de las organizaciones. México: McGraw-Hill; 2011.

13. Bertalanffy L. Teoria general de los sistemas. México, DF: Fondo de Cultura Económica; 2012.

14. Salanova $M$, Llorens $S$, Acosta $H$, Torrente P. Positive interventions in positive organizations. Ter Psicol. 2013; 31(1):101-113.

15. Asociación Española de Normalización y Certificación en México (AENOR). La integración de sistemas de gestión [en línea]. México DF: AENOR; 2010 [consultado 1 ene 2017]. Disponible en: http://www.aenormexico.com/certificación/ sistemas-integrados/

16. Diccionario de la lengua española. Madrid: Real Academia Española; 2014.

17. Universidad del Valle. Salud ocupacional: Factores de riesgo ocupacional [en línea]. Cali: Universidad del Valle; 2005 [consultado 10 oct 2016]. Disponible en: http://saludocupacional.univalle.edu.co/factoresderiesgoocupacionales.htm

18. RIMAC Seguros y Reaseguros. Indicadores de seguridad y salud en el trabajo (SST) [en línea]. Lima: RIMAC; 2007 [consultado 15 sep 2016]. Disponible en: http:// prevencionlaboralrimac.com/Herramientas/Indicadores-sst

19. Organización Internacional del Trabajo (OIT). Convención sobre los servicios de salud en el trabajo; Normlex Núm. 161 [en línea]. Ginebra: OIT; 1985 [consultado 15 jun 2016]. Disponible en: http://www.ilo.org/dyn/normlex/en/f?p=NOR MLEXPUB:12100:0::NO::P12100_ILO_CODE:C161

20. México. Secretaria del Trabajo y Previsión Social. Reglamento Federal de Seguridad y Salud en el Trabajo. Art. 3, frac. 15. Diario Oficial de la Federación [en línea] (13 Nov 2014); Tom. 734, No.9, p. 67-105 [consultado 15 sep 2016].
Disponible en: http://www.dof.gob.mx/nota_detalle.php?codigo=5368114\&f echa $=13 / 11 / 2014$

21. Oficina Internacional del Trabajo (OIT)- Organización Mundial de la Salud (OMS). Factores psicosociales en el trabajo. Informe del Comité Mixto OIT-OMS sobre Medicina del Trabajo [en linea] Ginebra: OIT-OMS; 1984 [consultado 10 oct 2016]. Disponible en: http://biblioteca.uces.edu.ar/MEDIA/EDOCS/FACTORES_Texto.pdf

22. México. Secretaría de Gobernación. Ley General de Protección Civil, Art. 2, frac. 41. Diario Oficial de la Federación [en línea]. Tom. 705, No. 4, 1a. Secc. (6 junio 2012]. [consultado 10 oct 2016]. p. 2-20. Disponible en: http://www.diputados. gob.mx/LeyesBiblio/pdf/LGPC_030614.pdf

23. Gobierno de México. Secretaria de Economía. Grandes empresas [en línea]. México DF: Secretaría de Economía; 2011. [consultado 10 oct 2016]. Disponible en: http://www.2006-2012.economia.gob.mx/mexico-emprende/empresas/granempresa

24. Escuela Europea de Excelencia. ISO 9001: Entendiendo el enfoque basado en procesos [en línea]. Santiago de Chile: EEE; 2014 [consultado 14 sep 2016]. Disponible en: http://www.nueva-iso-9001-2015.com/2014/11/iso-9001-entendiendo-enfoque-basado-procesos/

25. Anaya A. Manual de prácticas del Caso Integrador VI: Gestión integral de salud, seguridad, medio ambiente, protección civil, rescate y atención a emergencias. Guadalajara: Universidad de Guadalajara; 2015.

26. Escuela Europea de Excelencia. OHSAS 1800: Elaboración de una matriz IPER [en linea]. Santiago de Chile: EEE; 2014 [consultado 14 sep 2016]. Disponible en: http:// www.nueva-iso-45001.com/2014/12/ohsas-18001-elaboracion-matriz-iper

27. Anaya A. ¿Somos como trabajamos? La seguridad e higiene en el trabajo de pequeñas empresas de artesanías en un municipio del sur de Jalisco, México: una investigación acción participativa. Guadalajara: Universidad de Guadalajara; 2011.

28. Occupational Health and Safety Assessment Series (OHSAS). Norma OHSAS 18001:2007. Sistema de gestión en seguridad y salud ocupacional - Requisitos [en línea]. Londres: OHSAS Project Group; 2007 [consultado 15 dic 2016]. Disponible en: https://www.normasiso.net/wp-content/uploads/2016/02/ohsas-180012007.pdf

29. International Standard Organization (ISO). Norma ISO 9001:2015. Sistemas de gestión de la calidad - Requisitos [en línea]. Ginebra: ISO; 2015 [consultado 15 dic 2016]. Disponible en: https://www.iso.org/obp/ui/es/\#iso:std:iso:9001:ed5:v1:es

30. Organización Internacional del Trabajo (OIT). Directrices relativas a los sistemas de gestión de la seguridad y la salud en el trabajo, ILO-OSH 2001 [en línea]. Ginebra: OIT; 2002 [consultado 10 oct 2016]. Disponible en: http://www.ilo.org/safework/ info/standards-and-instruments/WCMS_112582/lang--es/index.htm

31. López F, Carrión MA. Aplicación de la metodologia de intervención psicosocial de la Asociación de Expertos en Psicosociología Aplicada (AEPA) en una empresa siderometalúrgica. InfoAEPA [en línea]. 2014 [consultado 15 dic 2016]; 10(15):15-27. Disponible en: http://openaccess.uoc.edu/webapps/o2/bitstream/ 10609/56224/1/L\%C3\%B3pez-Bar\%C3\%B3n_InfoAEPA2014_ Aplicaci\%C3\%B3n.pdf

\section{Referencias complementarias:}

Carrión MA. Batería para el estudio de las condiciones de trabajo de carácter psicosocial: CTCPS-MAC. Madrid: Académica Española; 2014.

International Standard Organization (ISO). Norma ISO 14001:2015. Sistemas de gestión ambiental - Requisitos con orientación para su uso [en línea]. Ginebra: ISO; 2015 [consultado 15 dic 2016]. Disponible en: https://www.iso.org/obp/ui/ es/\#iso:std:iso:14001:ed-3:v1:es

International Standard Organization (ISO). Norma ISO 22301:2012. Sistemas para la continuidad de negocio - Requisitos [en línea] 2012 [consultado 15 dic 2016]. Disponible en: https://www.iso.org/obp/ui/es/\#iso:std:iso:22301:ed-1:v2:en

Organización Internacional del Trabajo (OIT). Seguridad y salud en el trabajo [en línea]. Ginebra: OIT; 2016 [consultado 15 dic 2016]. Disponible en: http://www.lo.org/ global/topics/safety-and-health-at-work/lang--es/index.htm

Organización Internacional del Trabajo (OIT). Trabajo decente [en línea]. Ginebra: OIT; 2016 [consultado 10 oct 2016]. Disponible en: http://www.lo.org/global/topics/ decent-work/lang--es/index.htm 